\title{
GPSR-TARS: Congestion Aware Geographically Targeted Remote Surveillance for VANETs
}

\author{
Adam Walker, Milena Radenkovic \\ School of Computer Science \\ University of Nottingham \\ Jubilee Campus, Wollaton Road \\ Nottingham, NG8 1BB, UK \\ \{psyaw1,mvr\}@cs.nott.ac.uk
}

\begin{abstract}
Video over vehicular networks continues to receive warranted attention, with envisioned applications having the potential to present entirely new opportunities and revolutionise existing services. Many video systems have been proposed, ranging from safety to advertising. We propose a novel system for VANETs, namely the TArgeted Remote Surveillance (TARS) module for the existing Greedy Perimeter Stateless Routing (GPSR) protocol which permits multiple mobile vehicles to request and receive live video feeds from vehicles within a select geographic region. The multi-hop, vehicle-to-vehicle system enables mobile units to surveil a target area in real time by leveraging the dashboard cameras of vehicles moving within the target region. We combine several proposed extensions to the core protocol to introduce a dynamic real time congestion aware clustering scheme to achieve this. Our proposed system is compared against existing routing protocols using mobility data from Nottingham. GPSR-TARS outperforms the protocols assessed in key criteria crucial for meeting the quality of service demands of live multimedia dissemination.
\end{abstract}

\section{INTRODUCTION}

The benefits of on-demand video in vehicular networks are widely acknowledged, with recent research exploring many potential applications. One highly researched use case is for emergency services to access the video streams of cars at the scene of an accident, allowing them to make potentially life saving decisions before even arriving on the scene. Vehicular networks are continually advancing and moving forward we can expect vehicular clouds - sensor equipped, computationally capable connected vehicular networks which can pool resources - to become ever more integral to providing many services. It is acknowledged that the capabilities of vehicular clouds are underutilised and there are many open challenges in improving upon resource management at all layers [1].

Live multimedia streaming has strict requirements for proving quality experience to end users. These requirements largely revolve around delays and lost packets. Meeting these demands presents a significant challenge when such systems are deployed over decentralised networks. VANETs in particular raise their own issues with highly dynamic mobility forming a large portion of this challenge.

Aside from emergency video streams for traffic accidents, there is the potential for vehicle mounted cameras to augment the existing surveillance infrastructure in the investigation of crime or to provide monitoring in a region with no infrastructure, such as in military situations. Vehicles in an urban area move at speeds reasonable enough for a human observer to be able to make out key information from a video stream. To that end, we devise a targeted data requisition mechanism and congestion aware streaming module (TARS) and combine it with an existing position based routing protocol, namely GPSR, to permit mobile nodes to request and receive live data from all nodes within a select geographic region.

Though explored in a V2V network, it is the intent of this work to also build upon previous works in the field of sensor networks. The Fault and Disconnection Aware Smart Sensing (FDASS) framework [2] detects and mitigates the impact of malfunctions in sensor equipped devices in manufacturing environments whilst simultaneously providing select nodes with detailed notifications on the nature, location and time of any detected faults. Being able to gather live data on-demand from a select region of a manufacturing floor complements FDASS by leveraging devices in the vicinity of a detected fault to aid in the remote investigation of a malfunction. Thus, in addition to presenting an application for VANETs the proposed protocol simultaneously seeks to extend [2] by exploring novel functionality suitable for diverse sensor data. We focus our early exploration of GS PR-TARS on VANETs as vehicle mounted computers lack strict constraints on resources such as energy, memory capacity and computation, allowing us to largely focus on the congestion mitigation aspect of the system.

This paper takes the following format: related works are summarised in Section II; Section III describes the proposed GPSR-TARS system; in Section IV the setup of experiments and devised simulation scenarios is explained; Section V provides an evaluation of the results gathered in the previous section and in Section VI we provide concluding remarks.

\section{RELATED WORKS}

In recent years many video streaming protocols have been proposed for VANET specific applications each with varying foci. A range of approaches have been taken in providing performant, live video streams for a number of applications.

The V3 [3] system uses directed flooding to support streaming of video over a VANET from a single source to a large 
number of simultaneous receivers using velocity and location data to define forwarding zones in which elected nodes detect and update missing content in their neighbours' buffer. HIVE [4] is a performant video streaming protocol which has been shown to achieve high delivery ratios. VoV focuses supporting video streams in highly dynamic vehicular topologies [5], integrating features of delay tolerant networking. VIRTUS [6] is a unicast video streaming system which uses position, velocity and bearing to route video over the shortest, most stable link. LIAITHON builds upon [6] with route coupling prevention mechanisms. Video streaming protocols for VANETs have, with success, also been designed to intelligently and dynamically improve end user experience, e.g. [7]-[9].

Though finely tuned for video in $\mathrm{V} 2 \mathrm{~V}$ networks, on the whole the above protocols do not cope well with simultaneous encrypted video streams that mask any redundancy in duplicated data and do not easily permit meaningful optimisations to the stream. There is also a need to further explore the situation when multiple mobile senders and receivers are competing within the same area - only [8] and [3] are assessed with multiple video sources.

In the respective evaluations of the aforementioned protocols, typically sending and/or receiving node(s) are kept static. We therefore see a gap in the existing literature for a protocol for on-demand, high bandwidth data streams from multiple senders to multiple receivers.

Greedy Perimeter Stateless Routing (GPSR) [10] is a position based routing protocol which leverages the geographic position of nodes, detected through device mounted sensors such as GPS, to route traffic greedily through nodes with decreasing distance to the destination. In the case that a greedy path does not exist, GPSR uses it's backup perimeter mode to direct packets around the empty region until greedy forwarding can be resumed. Routing in GPSR is hop-byhop, with nodes only having knowledge of their immediate neighbours. Neighbour knowledge is built up through the receipt of periodic beacons which are broadcast to all nodes in range and contain the self reported position of a given host.

Several extensions have been proposed for GPSR. GPSR-M [11] improves upon the forwarding mechanism of the original protocol by factoring in node velocity. Nodes communicate their velocity in addition to their position, allowing a given node to detect which neighbours will move network traffic in an undesirable direction, such as could be the case were data distributed to vehicles in the opposing lane of a carriageway. E-GPSR [12], further improves upon the core protocol by adding proactive congestion avoidance through the routing of traffic away from nodes reaching buffer capacity. We combine the aforementioned extensions to GPSR and add our own congestion aware, geographic cluster module to to create GPSR-TARS.

\section{GPSR-TARS PROPOSAL}

We see the opportunity for a novel, cross layer system operating over vehicular clouds for supporting high-bandwidth, on-demand multimedia data from targeted regions for multiple

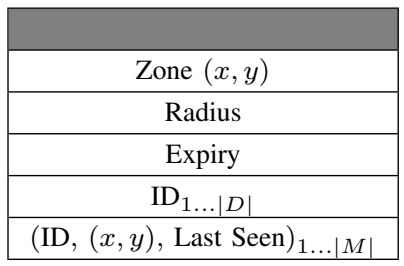

Fig. 1. Fields of HELLO+ Message

\begin{tabular}{|l|l|l|l|}
\hline Zone $(x, y)$ & Radius & Expiry & ID $_{d}$ \\
\hline
\end{tabular}

Fig. 2. Fields of SREQ Message

mobile receivers. GPSR-TARS aims to investigate how to improve quality of service in such a system, which can be a core part of the future vehicular cloud paradigm. We make no optimisations to the data stream and instead focus on protocol level performance, seeking to improve the experience of authorised surveilling nodes.

Our dynamic cluster scaling method reduces the size of the geographic area rather than selectively halting streams from nodes within the targeted region. Choosing the streams to drop would require providing the real time cluster with the ability to optimally calculate the minimum number of streams to provide maximal coverage of the surveillance area; although algorithms exist for this in positioning static surveillance cameras, calculating and dynamically coordinating the required parameters in an ad hoc network remains an open challenge. Outside of video, determining which nodes contain the most useful data for surveilling nodes whilst also minimising transmission of duplicate data would be a domain specific problem. This is beyond the scope of our project and thus we opt for dynamic radius scaling due to the requirement to keep overheads low.

Regarding the security of the system, PKI is employed to protect the streams from intermediary tampering and snooping as well as to identify the source of a stream should later investigation be required. We assume a that an authorised agency has generated a unique key pair for every car which is then stored in a mounted, tamper-proof hardware security module perhaps alongside hardcoded public keys for vehicles belonging to authorities with approved access to make surveillance requests. Vehicles around the world are already marked with unique a vehicle identification number (VIN) [13] and typically carry region specific license plates, the issuance of keys to vehicles could therefore be conducted as an extension of either of these existing services, with keys updated as part of a vehicle's mandatory periodic inspection.

Although it is possible for GPSR to operate in a purely reactive manner, we choose to retain the default, proactive system with nodes periodically distributing HELLO beacons to their immediate neighbours containing their position and, with the GPSR-M extension, vector. The overhead of broadcasting HELLO beacons is minimal and at the benefit of reduced routing time.

For our TARS module we augment the standard GPSR 


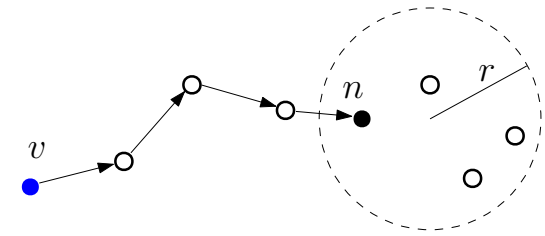

(a)

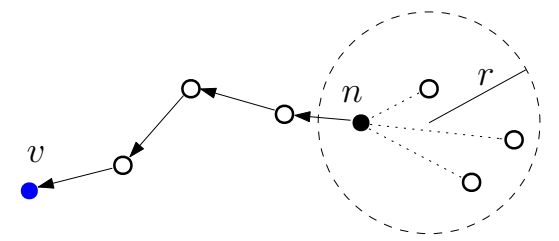

(b)

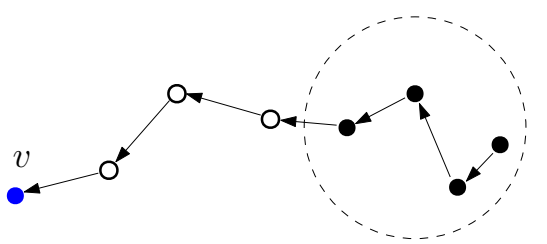

(c)

Fig. 3. Cluster formation process: (a) SREQ sent to region (b) SREQ received in region, streaming commenced by $n$ and HELLO+ messages distributed (c) All nodes in region streaming video to $v$

HELLO message with additional fields to create the HELLO+ beacon, which is broadcast by nodes within the surveillance region and contains: the aforementioned HELLO fields; the position and radius of the surveillance zone; the surveillance expiry time; the identities of nodes requesting surveillance; the identities and positions of nodes active in the zone with associated last-seen timestamps (Fig. 1). To determine which vehicles are in the region, nodes broadcast a list of nodes and their positions that they know to be in the region together with a timestamp of when they were last seen. This information is propagated in the area with removal of nodes from the list occurring if they haven't been observed in the last $5 \mathrm{~s}$.

To initiate surveillance an authorised vehicle, $v$, prepares and forwards a content-centric networking inspired surveillance request (SREQ) which is addressed to the target region rather than any specific node (Fig. 3a). The SREQ message contains the coordinates of the centre of the surveillance zone, the area's radius $(r)$, requisite identification information from the requesting node, and an expiry time (Fig. 1).

The first node in the target region to receive the SREQ, $n$, immediately begins providing a live video stream to $v$ and updates its HELLO+ beacon to include the necessary data from the SREQ, per Fig. 1 (Fig. 3b). Nodes already in, moving into, or exiting the surveillance zone who receive the HELLO+ beacon will propagate the data about the zone locally through their HELLO+ beacons so that streaming can begin and cease promptly (Figs. 3c and 4).

To alleviate congestion which may arise the cluster will dynamically adjust its size to best deliver traffic. It is in the interest of the emergency service vehicles to receive

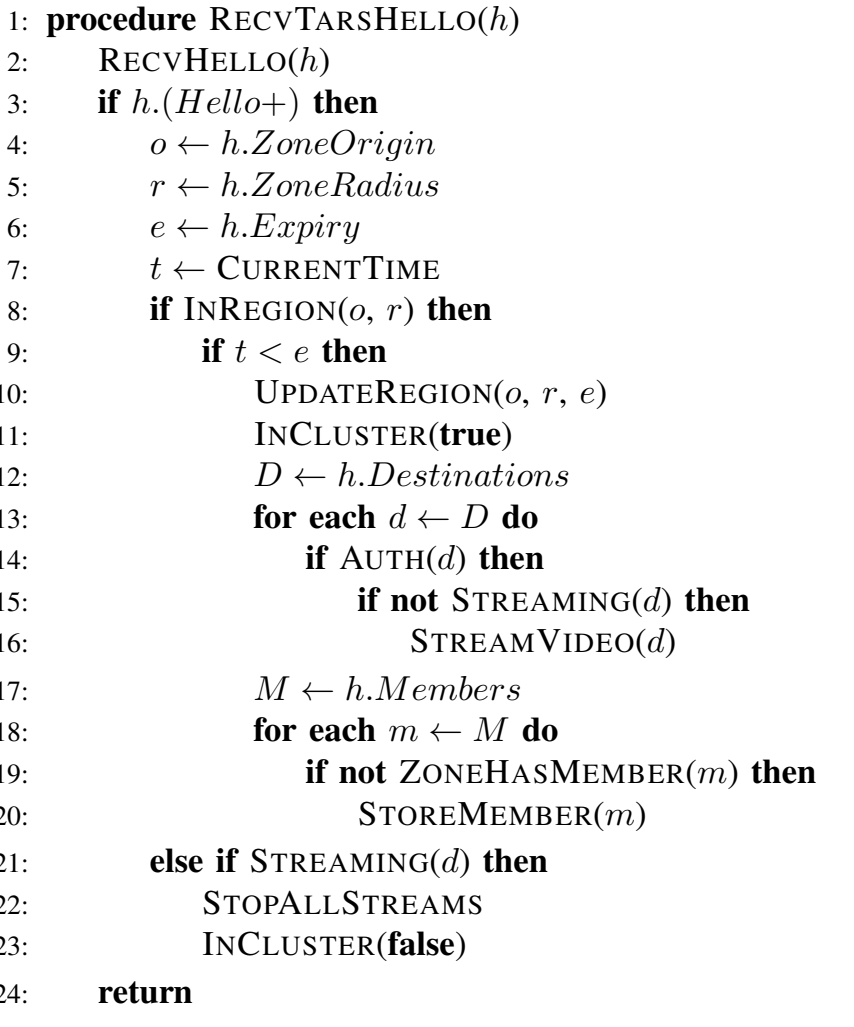

Fig. 4. HELLO+ Receiver Function

video streams from as close to the target as possible and so we implement cluster scaling by adjusting the radius of the surveillance zone. Congestion is detected by extending the EGPSR enhancement to report when a route becomes congested and packets are being rerouted. Whenever an alternate path is required to reduce the load on an existing route, a CONG message is sent to the cluster.

Upon receipt of a CONG message, the size of the surveillance region is scaled down to eliminate fringe nodes. In Fig. 5, congestion is detected between $a$ and $b$ and a single CONG message sent to the surveillance region; duplicate CONG messages are suppressed by intermediary nodes. Node $c$ the, first node in the cluster to receive the message, assumes temporary charge of the cluster and calculates the new radius which is then disseminated through the HELLO+ beacons. The new radius, $r_{i}$, of the surveillance zone is the previous radius reduced by a factor of the cluster density to promote radius reductions by greater increments in denser clusters, per (1). If multiple nodes have recalculated the radius then the latest, smallest radius is taken from the broadcast HELLO+.

The graph of all nodes within the surveillance region is represented by $G$, with $V$ selecting all vertices of a given graph; vertices exist between nodes regardless of network connection. The precise calculation of the eccentricity, $\epsilon$, of a vertex is dependent upon the coordinate system used - for GPS coordinates it would be necessary to use Vincenty's formulae [14]. 


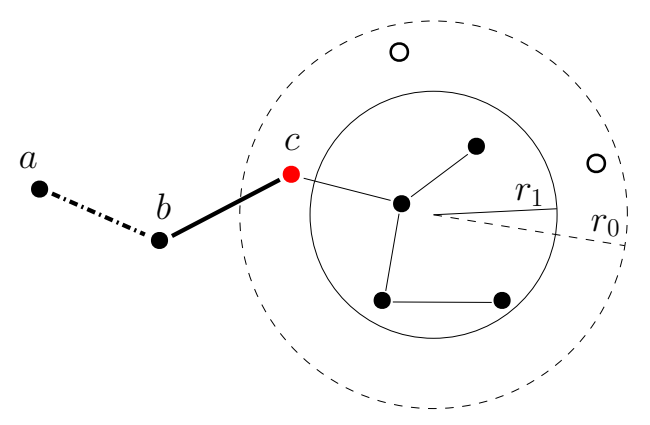

Fig. 5. Cluster scaling under congestion

TABLE I

SimUlation PARAMETERS

\begin{tabular}{|l|c|}
\hline Parameter & Value \\
\hline Simulator & $\mathrm{ns}-3.26$ \\
\hline MAC Layer & $802.11 \mathrm{p}$ \\
\hline Tx Power & $33 \mathrm{dBm}$ \\
\hline Propagation Loss Model & Nakagami \\
\hline Data Traffic & UDP, CBR at $2 \mathrm{Mbit} / \mathrm{s}$ \\
\hline Simulation Duration & $\sim 270 \mathrm{~s}$ \\
\hline Simulated Area & $5 \mathrm{~km} \times 5 \mathrm{~km}$, Nottingham, UK \\
\hline Civilian Nodes & $1,3,5$ \\
\hline Emergency Service Nodes & $<20 \mathrm{~m} / \mathrm{s}$ \\
\hline Civilian Node Speed & $<25 \mathrm{~m} / \mathrm{s}$ \\
\hline Emergency Node Speed &
\end{tabular}

$$
r_{i}=r_{i-1}+\frac{\alpha \cdot r_{i-1} \cdot|V(G)|}{\max _{v \in V} \epsilon(v)^{\beta}}
$$

In the absence of congestion, the surveillance zone is scaled up according to (2). As with (1), the density of the cluster determines the rate of cluster scaling but values for $\gamma$ and $\delta$ should be selected such that the increase in size occurs at a lower rate to avoid congestion collapse.

$$
r_{i}=r_{i-1}-\frac{\gamma \cdot r_{i-1} \cdot|V(G)|}{\max _{v \in V} \epsilon(v)^{\delta}}
$$

There is no message devised for remotely ending the surveillance prematurely and emergency vehicles entering the cluster participate in the system and distribute data from their dashcams to receivers outside of the zone until the expiry time has elapsed. If the requesting node wishes to extend the duration of the surveillance cluster then another request must be sent to the region and the expiry time updated accordingly. The cluster is dissolved once the requested duration of surveillance has passed.

\section{EXPERIMENT SETUP}

After initial early prototyping using the MODiToNeS [15] platform we embark upon further simulations using purely software approaches. GPSR-TARS is implemented in ns-3 [16], building upon existing work [11], [17]. To simulate an
TABLE II

GPSR-TARS CONFIGURATION

\begin{tabular}{|l|c|}
\hline Parameter & Value \\
\hline$\alpha$ & 6 \\
\hline$\beta$ & 1 \\
\hline$\gamma$ & 2 \\
\hline$\delta$ & 2 \\
\hline
\end{tabular}

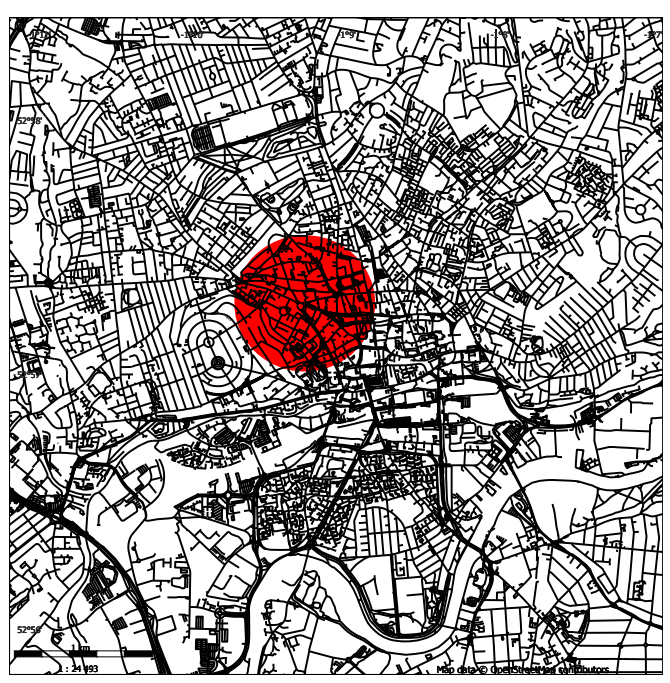

Fig. 6. $25 \mathrm{~km}^{2}$ area of Nottingham roads used in simulation with surveillance region highlighted

urban VANET, SUMO [18] was used to generate realistic vehicular traffic from a $5 \mathrm{~km} \times 5 \mathrm{~km}$ map of Nottingham, UK (Fig. 6) exported from OpenStreetMap. The rulesets used to create traces were adjusted to account for local traffic regulations such as speed restrictions. Random traces were generated for civilian vehicles and combined with traces for emergency service vehicles which were configured to start at the edge of the map and move towards the surveillance zone. Traces were exported to an ns-3 compatible TCL format which mapped GPS coordinates to points on a two-dimensional Cartesian plane, the eccentricity of any vertex is thus calculated using the Pythagorean theorem.

Vehicles are equipped with 802.11 p with the proposed system operating in the reserved service channel. The simulation of encrypted video traffic is accomplished by nodes transmitting constant bit-rate data at $2 \mathrm{Mbit} / \mathrm{s}$. No infrastructure nodes are included in the scenario.

A desired surveillance region of $500 \mathrm{~m}$ radius centred at $52^{\circ} 57^{\prime} 15.4152^{\prime \prime},-1^{\circ} 9^{\prime} 18.8568^{\prime \prime}$ (highlighted in Fig. 6) is requested by emergency vehicles. The location of the surveillance zone allows us to evaluate the performance of the protocol as emergency vehicles, positioned on the edge of the map, are en route to the situation with decreasing distance to the target area. The surveillance region itself contains a number of different road categories ranging from slower single lane one-way streets to faster dual carriageways.

Emergency vehicles in the scenario are initially positioned 
at random locations on the fringe of the city and move towards the centre of the surveillance region. Once movement has commenced, the emergency vehicles begin sending their requests for surveillance. The simulation ceases once all emergency vehicles have reached the zone. Emergency service vehicles move towards the surveillance zone faster than other nodes but obey all other road regulations.

We run the scenario with incrementing numbers of emergency service vehicles to explore adaptation to congestion. For all data gathered the results represent the average of 10 runs of the scenario. Comparisons are made against AODV [19], DSDV [20], and the raw E-GPSR-M protocol. To ensure these comparisons are fair both protocols are modified to have the ability to broadcast the necessary data required to inform others of the surveillance region, following receipt of an SREQ.

\section{Simulation Results}

Performance analysis of GPSR-TARS is focused on the quality of service needs of live multimedia. Because of the time sensitive nature of video it is vital that we assess the system according end-to-end delay and packet loss as lost data and high delays lead to significant degradation in end user perceived quality

There is no difference between GPSR-TARS and GPSR in terms of packet delivery when there is only 1 receiving node, owing to the fact that no scaling of the surveillance zone is undertaken (Fig. 7) however with increasing numbers of receiving nodes congestion becomes more challenging. We show that the delivery ratio drops for both protocols but that our TARS module allows better recovery than the standard protocol by almost $10 \%$ when there is more than a single emergency service node active in the scenario. The dynamic scaling of the region evens out the loss incurred by congestion when there are 5 receiving nodes while the keeping the packet loss below $7 \%$. Interestingly, and as has been observed by others investigating urban VANETs [21], AODV maintains a higher delivery ratio than the greedy protocols and DSDV at all numbers of receiving nodes.

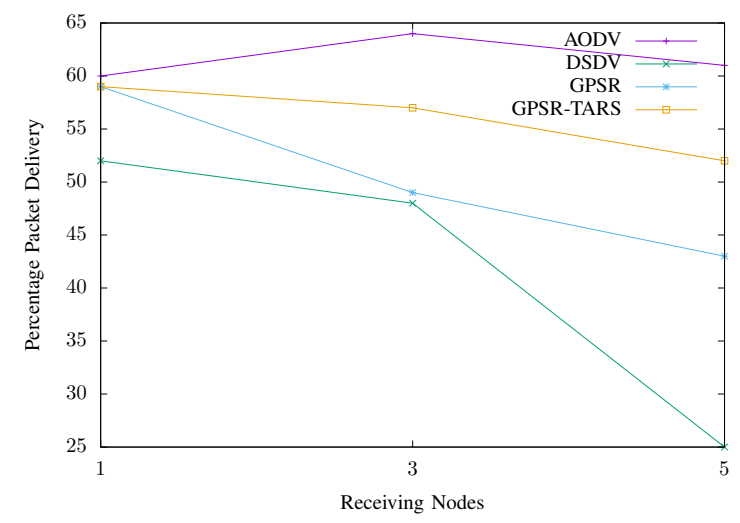

Fig. 7. PDR at increasing surveillance nodes
GPSR-TARS outperforms all other analysed protocols in terms of end-to-end delay at all numbers of emergency service nodes tested. At 5 receiving nodes the delays incurred through GPSR-TARS remain lower than for AODV at a single receiver. Furthermore, GPSR-TARS sees delays even-out when compared to the original protocol. As nodes within the surveillance region respond to congestion the scaling keeps delays to a minimum, demonstrating a lower rate of increase than other protocols.

With the increase in data flowing through the network to additional receivers the cluster must dynamically adjusts its size to reduce the impact of congestion. Without adaptation all nodes in the region transmit video (Fig. 9a) with no consideration to the effect of increasing data. The PKI system deployed mandates unique keys for each emergency service vehicle therefore the system cannot support either intelligent buffer management through selectively ignoring duplicate data or the source node addressing identical data to multiple receivers. Our module is effective at improving performance because the number of active nodes adapts to the requirements of the network (Fig. 9a) albeit at the trade-off of reduced coverage of the target zone.

\section{CONCLUSION}

This work has explored GPSR-TARS, a novel system for targeted, on-demand data collection which we evaluated using a VANET scenario. The scenario devised was assessed with multiple, mobile sending and receiving nodes at varying quantities of each. GPSR-TARS demonstrated improved performance over several existing protocols, most crucially in the areas directly related to end user quality of service.

In our future work we will give greater consideration to protocol overheads and resource constraints so that the system can be targeted to nodes with stricter requirements. In a location-targeted data collection system supporting heterogeneous nodes it could be expected that a portion of the network would consist of battery powered devices and/or have limited computational ability. We will therefore investigate proposals such as [22] to enable the system to be more resource considerate and disconnection tolerant.

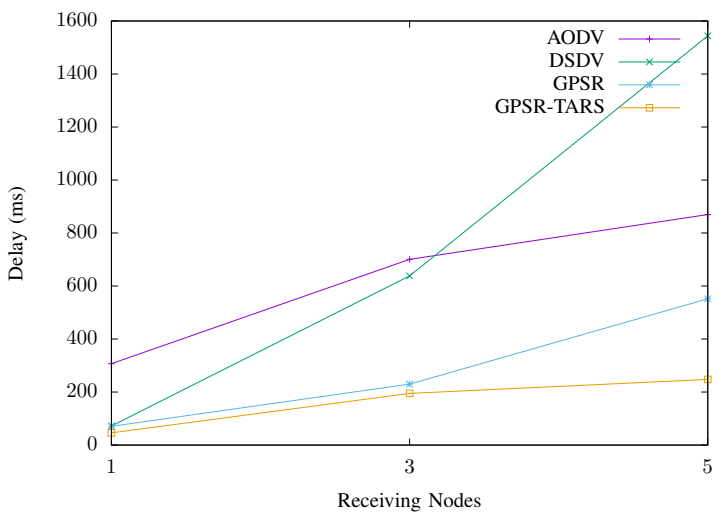

Fig. 8. Delay at increasing surveillance nodes 


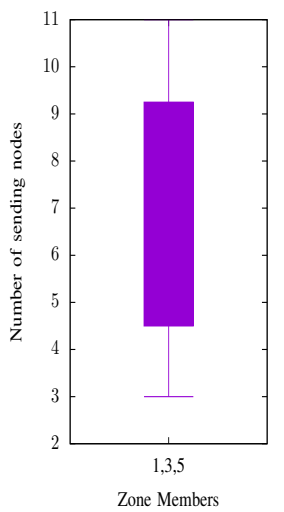

(a)

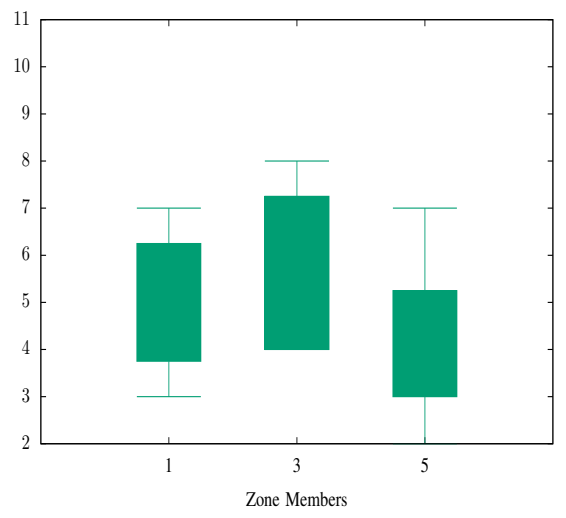

(b)
Fig. 9. Active streaming nodes during simulation at increasing surveilling nodes for (a) AODV, DSDV \& GPSR (b) GPSR-TARS

Our TARS module is to be enhanced with features of other position based routing protocols to improve on performance over distance; in particular Connectivity Aware Routing (CAR) [23] will be looked at as the route preserving guard nodes could improve on the overall delivery ratio. Support will also be added for the moving and merging of live clusters. In a future project we would like to flesh out the content aspect of the system and add support for requesting location specific historic data to gather information about an event that has already occurred. For example, vehicles that were active in a target area at some point in time could provide crucial evidence of an incident and facilitate retroactive investigation.

Moving beyond simulated scenarios, for further realism future prototypes are to be assessed using the MODiToNeS testbed [15], which uses Raspberry Pis to create a versatile platform for assessing protocols with high realism whilst simultaneously providing support for extensive real-time data analysis. This will be deployed in real vehicles in Nottingham.

\section{ACKNOWLEDGMENT}

This work was supported in part by the Project Health Monitoring and Life-Long Capability Management for SELfSUStaining Manufacturing Systems through the Commission of the European Communities under the 7th Framework Programme, under Grant 609382.

\section{REFERENCES}

[1] T. Mekki, I. Jabri, A. Rachedi, and M. ben Jemaa, "Vehicular cloud networks: Challenges, architectures, and future directions," Vehicular Communications, pp. -, 2016.

[2] M. Radenkovic, I. Kostadinov, and B. Wietrzyk, "Increasing communication reliability in manufacturing environments," in 2015 International Wireless Communications and Mobile Computing Conference (IWCMC), Aug 2015, pp. 1377-1383.

[3] M. Guo, M. H. Ammar, and E. W. Zegura, "V3: a vehicle-to-vehicle live video streaming architecture," in Third IEEE International Conference on Pervasive Computing and Communications, March 2005, pp. 171180.

[4] F. Naeimipoor and A. Boukerche, "A hybrid video dissemination protocol for vanets," in 2014 IEEE International Conference on Communications (ICC), June 2014, pp. 112-117.
[5] "A rate control video dissemination solution for extremely dynamic vehicular ad hoc networks," Perform. Eval., vol. 87, no. C, pp. 3-18, May 2015.

[6] C. Rezende, H. S. Ramos, R. W. Pazzi, A. Boukerche, A. C. Frery, and A. A. F. Loureiro, "Virtus: A resilient location-aware video unicast scheme for vehicular networks," in 2012 IEEE International Conference on Communications (ICC), June 2012, pp. 698-702.

[7] C. Rezende, A. Boukerche, M. Almulla, and A. A. Loureiro, "The selective use of redundancy for video streaming over vehicular ad hoc networks," Computer Networks, vol. 81, pp. 43 - 62, 2015.

[8] C. Quadros, A. Santos, M. Gerla, and E. Cerqueira, "A qoe-aware mechanism to improve the dissemination of live videos over vanets," in Computer Networks and Distributed Systems (SBRC), 2015 XXXIII Brazilian Symposium on, May 2015, pp. 31-40.

[9] C. Quadros, E. Cerqueira, A. Santos, J. Lim, and M. Gerla, "Beacon-less video streaming management for vanets based on qoe and link-quality," in 2015 IFIP/IEEE International Symposium on Integrated Network Management (IM), May 2015, pp. 191-198.

[10] B. Karp and H. T. Kung, "Gpsr: Greedy perimeter stateless routing for wireless networks," in Proceedings of the 6th Annual International Conference on Mobile Computing and Networking, ser. MobiCom '00. New York, NY, USA: ACM, 2000, pp. 243-254.

[11] C. Bouras, V. Kapoulas, and E. Tsanai, A GPSR Enhancement Mechanism for Routing in VANETs. Cham: Springer International Publishing, 2015, pp. 94-107.

[12] T. Hu, M. Liwang, L. Huang, and Y. Tang, "An enhanced gpsr routing protocol based on the buffer length of nodes for the congestion problem in vanets," in 2015 10th International Conference on Computer Science Education (ICCSE), July 2015, pp. 416-419.

[13] ISO, "Road vehicles - types - terms and definitions," International Organization for Standardization, Geneva, Switzerland, ISO 3833:1997, 1997.

[14] T. Vincenty, "Direct and inverse solutions of geodesics on the ellipsoid with application of nested equations," Survey Review, vol. 23, no. 176, pp. 88-93, 1975.

[15] M. Radenkovic, J. Crowcroft, and M. H. Rehmani, "Towards low cost prototyping of mobile opportunistic disconnection tolerant networks and systems," IEEE Access, vol. 4, pp. 5309-5321, 2016.

[16] G. F. Riley and T. R. Henderson, The ns-3 Network Simulator. Berlin, Heidelberg: Springer Berlin Heidelberg, 2010, pp. 15-34.

[17] A. Fonseca, A. Camões, and T. Vazão, "Geographical routing implementation in ns3," in Proceedings of the 5th International ICST Conference on Simulation Tools and Techniques, ser. SIMUTOOLS '12. ICST, Brussels, Belgium, Belgium: ICST (Institute for Computer Sciences, Social-Informatics and Telecommunications Engineering), 2012, pp. 353-358.

[18] D. Krajzewicz, J. Erdmann, M. Behrisch, and L. Bieker, "Recent development and applications of SUMO - Simulation of Urban MObility," International Journal On Advances in Systems and Measurements, vol. 5, no. 3\&4, pp. 128-138, December 2012.

[19] C. Perkins, E. Royer, and S. Das, "Rfc 3561 ad hoc on-demand distance vector (aodv) routing," Tech. Rep., 2003. [Online]. Available: http://tools.ietf.org/html/rfc3561

[20] C. E. Perkins and P. Bhagwat, "Highly dynamic destination-sequenced distance-vector routing (dsdv) for mobile computers," in Proceedings of the Conference on Communications Architectures, Protocols and Applications, ser. SIGCOMM '94. New York, NY, USA: ACM, 1994, pp. 234-244.

[21] R. Bala and C. R. Krishna, "Scenario based performance analysis of aodv and gpsr routing protocols in a vanet," in 2015 IEEE International Conference on Computational Intelligence Communication Technology, Feb 2015, pp. 432-437.

[22] R. Alsaqour, M. Abdelhaq, R. Saeed, M. Uddin, O. Alsukour, M. AlHubaishi, and T. Alahdal, "Dynamic packet beaconing for gpsr mobile ad hoc position-based routing protocol using fuzzy logic," J. Netw. Comput. Appl., vol. 47, no. C, pp. 32-46, Jan. 2015.

[23] Q. Yang, A. Lim, and P. Agrawal, "Connectivity aware routing in vehicular networks," in 2008 IEEE Wireless Communications and Networking Conference, March 2008, pp. 2218-2223. 